\title{
Endogenous methyl palmitate modulates nicotinic receptor-mediated transmission in the superior cervical ganglion
}

\author{
Hung Wen Linn, \\ Alex F. Chen ${ }^{h}$, Louis S. Premkumara, Donald S. Torry', and Tony J.-F. Lee ${ }^{a, c, d, e, 1}$ \\ Departments of aPharmacology and 'Medical Microbiology, Immunology, and Cell Biology, Southern Illinois University School of Medicine, Springfield, IL \\ 62702: dCenter for Vascular Medicine, College of Life Sciences and 'Departments of Pharmacology and Neuroscience, College of Medicine, Tzu-Chi

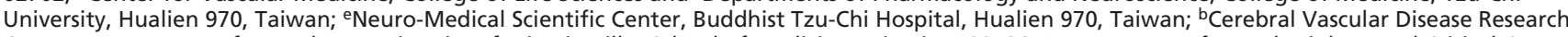 \\ Center, Department of Neurology, University of Miami, Miller School of Medicine, Miami, FL 33136; 9Department of Anesthesiology and Critical Care \\ Medicine, The Johns Hopkins University, Baltimore, MD 21205; hDepartment of Pharmacology and Toxicology, Michigan State University, \\ East Lansing, MI 48824; and ${ }^{f}$ Center for Neuropsychiatry, China Medical University and Hospital, Taichung 406, Taiwan
}

Communicated by Salih J. Wakil, Baylor College of Medicine, Houston, TX, October 14, 2008 (received for review June 23, 2008)

Nitric oxide (NO) is identified as the endothelium-derived relaxing factor and a neurotransmitter with a superfusion bioassay cascade technique. By using a similar technique with rat superior cervical ganglion (SCG) as donor tissue and rabbit endothelium-denuded aortic ring as detector tissue, we report here that a vasodilator, which is more potent than NO, is released in the SCG upon field electrical stimulation (FES) or addition of nicotine. Release of this vasodilator was enhanced by arginine analogs, including $N^{\omega}{ }^{\omega}$-nitro-L-arginine (a NO synthase inhibitor), suggesting that it is not NO. Analysis by gas chromatography/mass spectrometry identified 2 saturated fatty acids, palmitic acid methyl ester (PAME) and stearic acid methyl ester (SAME), being released from the SCG upon FES in the presence of arginine analogs. Exogenous PAME but not SAME induced significant aortic dilation $\left(E_{50}=0.19 \mathrm{nM}\right)$, indicating that PAME is the potent vasodilator. Release of PAME and SAME was significantly diminished in chronically decentralized SCG but not denervated SCG, suggesting the preganglionic origin. Furthermore, release of both fatty acids was calcium- and myosin light chain kinase-dependent, suggesting that both were released from axoplasmic vesicular stores. Electrophysiological studies further demonstrated that PAME but not SAME inhibited nicotine-induced inward currents in cultured SCG and the $\alpha 7$-nicotinic acetylcholine receptor-expressing Xenopus oocytes. Endogenous PAME appears to play a role in modulation of the autonomic ganglionic transmission and to complement the vasodilator effect of NO.

alpha7-nicotinic acetylcholine receptor | fatty acid vasodilator | palmitic and stearic acid methyl ester | superfusion bioassay cascade

$t$ is well established that cerebral blood vessels of several species receive sympathetic innervation originating in the superior cervical ganglion (SCG). The functional role of this innervation, however, has not been completely elucidated (1-3). Even though a reasonable knowledge of the transmission mechanisms at the neurovascular synapses is available (1-3), the ganglionic transmission at the SCG is far less understood. Nitric oxide synthase (NOS) immunoreactivities have been demonstrated in strands of nerve fibers entering the rat SCG (4), suggesting that NO (5) plays a role in sympathetic ganglionic transmission (6). Accordingly, we examined whether NO was released upon depolarization of the SCG neurons, by using a superfusion bioassay cascade system $(7,8)$ with the rat SCG as donor tissue and rabbit endothelium-denuded aortic ring (e-RbA) as detector tissue (8).

\section{Results and Discussion}

Field electrical stimulation (FES) at 4, 8, and $16 \mathrm{~Hz}$ of the SCG resulted in frequency-dependent dilation of the e-RbA precontracted with L-phenylephrine $(0.1 \mu \mathrm{M})$ (Fig. $1 A)$. The dilation was significantly enhanced for each stimulation frequency $(n=3-8)$ (Fig. $1 B$ and $C$ ) upon addition of $N^{\omega}$-nitro-L-arginine (NLA, 0.3 $\mathrm{mM}$ ) and $N^{\omega}$-nitro-L-arginine methyl ester (L-NAME, $0.3 \mathrm{mM}$ ) onto the SCG, while these inhibitors alone did not change the basal tone of the e-RbA. Furthermore, this vasodilation was not affected in the presence of a NO scavenger 2-(4-carboxyphenyl)-4,4,5,5tetramethylimidazoline-1-oxyl-3-oxide potassium salt (carboxylPTIO, $30 \mu \mathrm{M}$ ) (data not shown). These results suggest release of a non-NO transferable vasodilator substance (TVS) from the SCG upon depolarization. The FES-of-the SCG-induced aortic dilation also was enhanced by $N^{\omega}$-nitro-D-arginine (NDA, $0.3 \mathrm{mM}$, not a NOS inhibitor; Fig. $1 D$ ), L-arginine (L-Arg), or D-arginine (D-Arg) $(0.3 \mathrm{mM})$ (Fig. $1 E$ and $F)$ superfused onto the SCG. These arginine analogs are known to enter the cell, which either promote or disrupt the conversion of L-arginine to L-citrulline, a major pathway in the production of NO (9). Because addition of these arginine analogs did not result in additive vasodilatory effects of the e-RbA, these analogs most likely act in a similar manner to enhance FES-induced TVS release in the SCG. Furthermore, FES-of-the-SCG-induced aortic dilation was enhanced by L-lysine (L-Lys), D-lysine (D-Lys), and L-histidine (L-His) $(0.3 \mathrm{mM})$, but not by L-phenylalanine (L-Phe) $(0.3 \mathrm{mM})$ superfused onto the SCG (Fig. $1 G)$, suggesting modulation of TVS release by certain amino acids.

Subsequent studies using pharmacological antagonists show that the TVS is nonadrenergic and noncholinergic and is not carbon monoxide (CO), vasoactive intestinal peptide (VIP), calcitonin gene-related peptide (CGRP), or epoxyeicosatrienoic acids (EET) (Fig. $1 J-M$ ). Biochemical analyses by reverse-phase high-performance liquid chromatography (RP-HPLC) of the superfusates collected following superfusing the SCG and e-RbA demonstrated that superfusion of Krebs' only, Krebs' plus FES of the SCG, and Krebs' containing NLA onto the SCG resulted in many small peaks (Fig. 2A). Two particular small peaks with retention times of $23.760 \mathrm{~min}$ (peak 1/P1) and 27.153 $\min$ (peak 2/P2) following application of NLA $(0.3 \mathrm{mM})$ onto the SCG were significantly enhanced upon FES of the SCG (Fig. 2A). These 2 peak samples were individually collected, lyophilized, reconstituted, and reapplied onto the e-RbA. Only $\mathrm{P} 2$ sample induced significant aortic relaxation with a time course similar to that of the aortic relaxation induced by TVS upon FES of the SCG in the presence of NLA (Fig. 2B).

Author contributions: H.W.L., C.-Z.L., D.C., P.-Y.C., M.-F.C., S.-Z.L., M.M., A.F.C., L.S.P., D.S.T. and T.J.-F.L. designed research; H.W.L., C.-Z.L., D.C., P.-Y.C., and T.J.-F.L. performed re search; H.W.L., C.-Z.L., D.C., P.-Y.C., M.-F.C., L.S.P., D.S.T., and T.J.-F.L. contributed new reagents/analytic tools; H.W.L., C.-Z.L., D.C., P.-Y.C., M.-F.C., A.F.C., L.S.P., D.S.T., and T.J.-F.L. analyzed data; and H.W.L., C.-Z.L., M.-F.C., and T.J.-F.L. wrote the paper.

The authors declare no conflict of interest.

Freely available online through the PNAS open access option.

${ }^{1}$ To whom correspondence should be addressed. E-mail: tlee@mail.tcu.edu.tw.

This article contains supporting information online at www.pnas.org/cgi/content/full/ 0810262105/DCSupplemental.

๑ 2008 by The National Academy of Sciences of the USA 

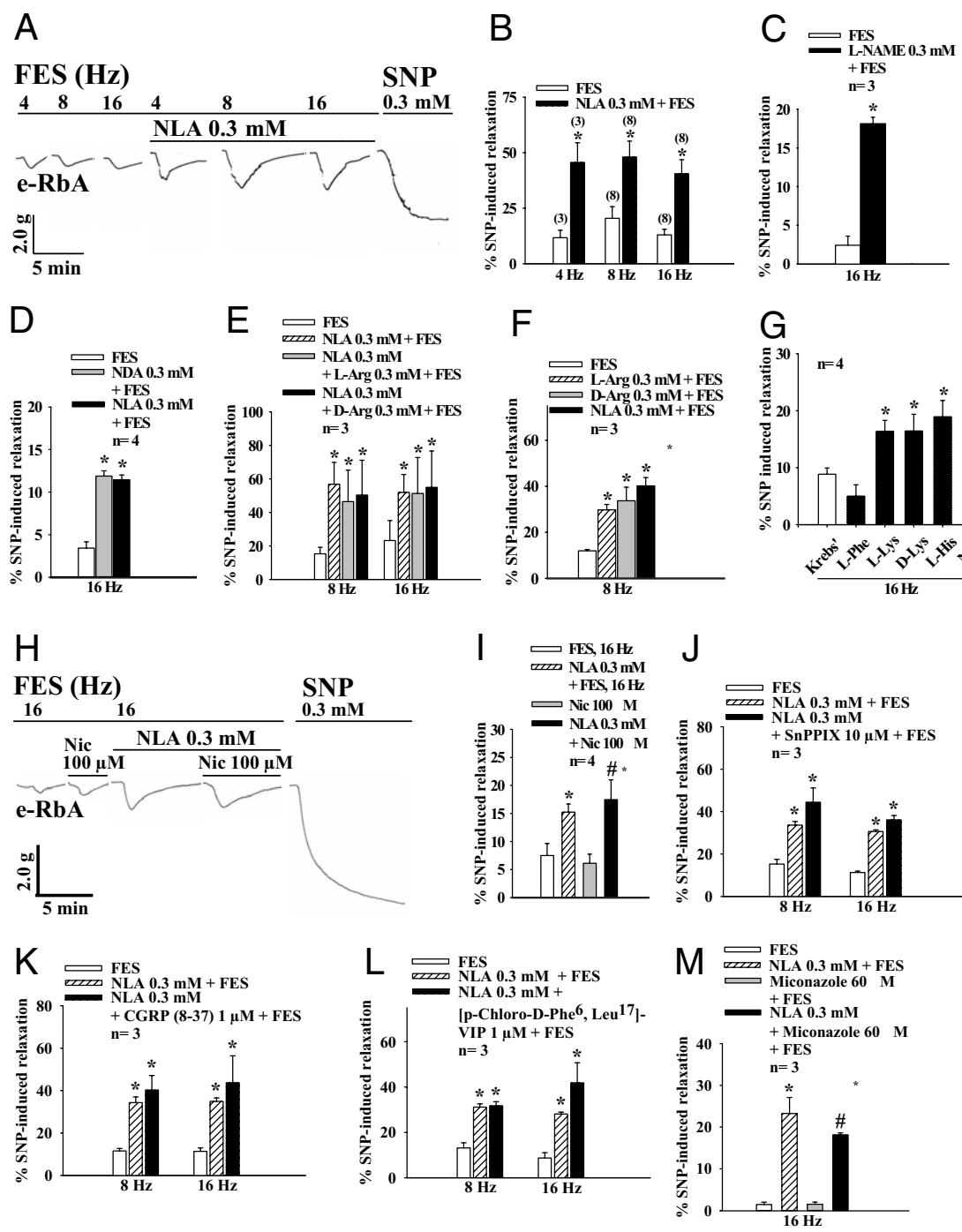

Fig. 1. Dilation of the e-RbA (the detector tissue) upon depolarization of the SCG (the donor tissue) by FES $(A-G)$ or application of nicotine $(H$ and $I)$ onto the SCG was enhanced by arginine analogs and related amino acids, but not by SnPPIX, CGRP, or VIP receptor antagonist, or miconazole $(J-M)$ superfused over the SCG. $(A)$ Representative tracing showing aortic dilation upon FES at different frequencies $(4,8$, and $16 \mathrm{~Hz})$ in the presence or absence of NLA (0.3 mM) superfused over the SCG, and the effects of NLA are summarized in $B$. Other experimental drugs superfused directly onto the SCG enhanced FES-of-the-SCG-induced aortic relaxation including $(C)$ L-NAME (0.3 mM) (D) NDA (0.3 mM) followed by NLA, $(E)$ NLA (0.3 mM) followed by L-Arg or D-Arg (0.3 mM), (F) L-Arg or D-Arg (0.3 mM), and (G) L-Lys, D-Lys, L-His, or NLA (0.3 mM). In contrast, L-phenylalanine (Phe) (0.3 mM) (G) did not affect aortic relaxation. $(H)$ Representative tracing showing that nicotine (Nic, $100 \mu \mathrm{M}$ ) superfused directly over the SCG resulted in aortic dilation that was significantly enhanced by NLA superfused over the SCG. This latter effect is summarized in I. As a negative control, nicotine applied directly onto e-RbA did not cause relaxation in the presence or absence of NLA. ( $)$ SnPPIX (10 $\mu \mathrm{M}$, a HO-1 competitive inhibitor), (K) CGRP 8-37 (1 $\mu \mathrm{M}$, a CGRP receptor antagonist), $(L)$ [p-chloro-D-Phe ${ }^{6}$, Leu $\left.{ }^{17}\right]$ VIP (1 $\mu \mathrm{M}$, a VIP receptor antagonist), and $(M)$ miconazole ( $60 \mu \mathrm{M}$, an EET synthesis inhibitor) superfused over the SCG in the presence of NLA $(0.3 \mathrm{mM})$ did not affect FES-of-the-SCG-induced dilation of the e-RbA in the presence of active muscle tone induced by L-phenylephrine $(0.1 \mu \mathrm{M})$. SNP $(0.3 \mathrm{mM})$ was given to induce maximal relaxation at the end of each experiment. *, $P<0.05$ denotes significantly different from FES only, and \#, $P<$ 0.05 indicates significantly different from miconazole plus FES or nicotine only. $n$ denotes the number of experiments.
The P2 samples were subjected further to gas chromatography/ mass spectrometry (GC/MS) analysis and resulted in 2 peaks at 24 min and $26.5 \mathrm{~min}$ of retention time (Fig. $3 A$ ). Similar GC/MS profiles in the superfusates of the bioassay cascade without purification by RP-HPLC were obtained. Comparison of the 2 peaks with the MS library suggests that the peak sample at 24 min having a molecular weight $\left(M_{\mathrm{r}}\right)$ of 270 (Fig. $\left.3 B\right)$ is palmitic acid methyl ester (PAME) and that at 26.5 min having $M_{\mathrm{r}}$ of 298 is stearic acid methyl ester (SAME) (Fig. 3B). For control, heptadecanoic acid 16 methyl, methyl ester, which has similar $M_{\mathrm{r}}$ as SAME, produced different retention time (data not shown).

Consistent with aortic relaxation responses, superfusion of NLA (Fig. 3A), L-Arg (Fig. 3C), and D-Arg (Fig. 3D), but not L-Phe (Fig. $3 E)$, significantly enhanced FES-of-the-SCG-induced release of PAME and SAME without affecting arachidonic acid release, suggesting that the release of PAME and SAME is a viable biological mechanism rather than an artifact (Fig. 3A). The selective enhancement of PAME and SAME release may suggest that both fatty acid methyl esters (FAMEs) are potential TVS. Nonetheless, exogenous PAME (1 pM-0.1 $\mu \mathrm{M})($ Fig. $3 F$ and $G$ ) but not SAME (Fig. $3 G$ ), stearic acid (SA), or palmitic acid (PA) (Fig. 3H) applied directly onto the e-RbA produced concentration-dependent aortic dilation with geometric mean $\mathrm{EC}_{50}$ values $(10)$ of $1.92(0.46-7.93) \times$ $10^{-10} \mathrm{M}$ and with a time course (Fig. $3 F$ ) similar to relaxation induced by the TVS released from the SCG upon FES in the presence of NLA (Fig. 1A). This finding that PA does not cause vasodilation is similar to that reported in the rat basilar arteries (11). These results suggest that PAME but not SAME, SA, or PA is the TVS. Furthermore, the maximal release of PAME from the SCG upon FES at $16 \mathrm{~Hz}$ was $0.37 \mu \mathrm{M}$ based on the known volume of experimental solution and concentration of standard PAME solution of $0.1 \mu \mathrm{g} / \mathrm{mL}$ injected in the GC/MS, and the $M_{\mathrm{r}}$ of PAME. This micromolar concentration of endogenous PAME is consistent with the concentration of exogenous PAME that induces maximum vasodilation as shown in Fig. $3 G$. This finding further supports that PAME is the TVS. The possible involvement of other bioactive compounds with short half-lives that were not detected because of technical limitations of the GC/MS, however, cannot be excluded.

The geometric mean $\mathrm{EC}_{50}$ value for sodium nitroprusside (SNP, a NO donor) in inducing aortic relaxation in the present work is 7.44(4.52-12.2) $\times 10^{-7} \mathrm{M}(n=4)$. Accordingly, PAME is 3,000 times more potent than SNP. PAME also is more potent than SNP in different tissues (12) and other vasodilators such as PROLI/NO (13), spermine NONOate (14), adenosine $(15,16)$, EET $(17,18)$, CO (19), VIP $(20,21)$, and CGRP $(20,22)$, suggesting that PAME is the most potent known vasodilator. The exact mechanism for PAME-induced vasodilation remains to be determined. Esterization of PA appears to be important for its dilator response based on the presenting findings. Significant release of PAME following inhibition of NO synthesis by arginine analogs suggests that PAME may complement vasodilator 
A

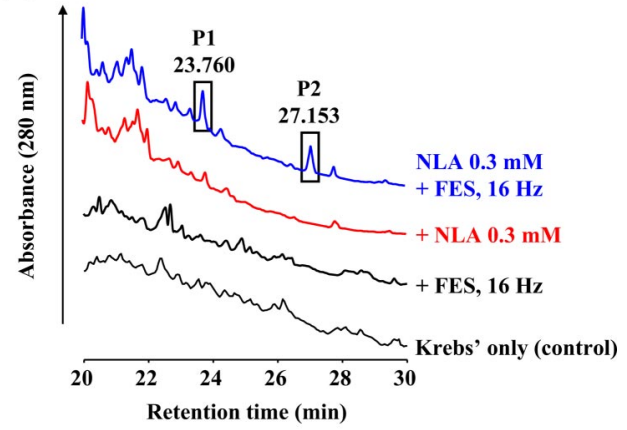

$\mathrm{B}$

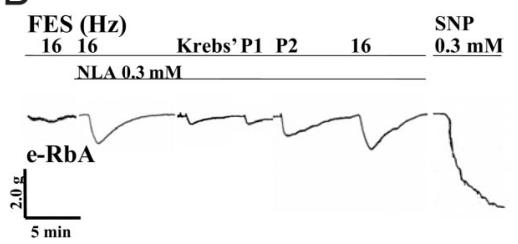

Fig. 2. Absorbance peak containing the transferable vasodilator substance. $(A)$ Absorbance peaks of a superfusion sample collected following superfusing the SCG with Krebs' solution (control), FES of the SCG, NLA $(0.3 \mathrm{mM})$ alone, or after FES of the SCG in the presence of NLA. Two particular peaks at 23.760 min (peak $1 / \mathrm{P} 1$ ) and $27.153 \mathrm{~min}$ (peak 2/P2) that were significantly increased are highlighted in vertical rectangles. $(B)$ Each peak was collected, lyophilized, reconstituted in 60 $\mu \mathrm{L}$ of Krebs' solution, and reapplied directly onto the e-RbA in the presence of NLA (0.3 mM). Peak 1 (P1) fraction caused basal aortic dilation that was equivalent to that induced by Krebs' solution containing NLA only, suggesting that P1induced relaxation is not an active chemical-induced dilation. Peak 2 (P2) fraction, however, caused significant aortic dilation. The time course of peak 2 fractioninduced relaxation was similar to that induced by the TVS from the SCG upon FES in the presence of NLA. In the absence of NLA, FES of the SCG caused a very small or no relaxation ( $n=2$ for all experiments). FES-of-the-SCG-induced relaxation in the presence of NLA $(0.3 \mathrm{mM}, 60 \mu \mathrm{L})$ superfused over the e-RbA served as control.

response of NO. Furthermore, the SCG is a highly vascularized organ (23). Factors involved in regulating the vascular function and blood flow in the SCG are largely unknown. Our present findings suggest that PAME released locally at the SCG is expected to cause vasodilation and increase the circulation in this ganglion.

To determine the origin of PAME and SAME in the SCG, release of both from the SCG 5 days after surgical transection of pre-SCG sympathetic nerves [decentralization; leading to degeneration of the preganglionic nerve terminals of the SCG $(2,4)]$ or post-SCG sympathetic nerves [denervation; leading to degeneration of the SCG cell bodies and postganglionic neurons $(2,24)]$ was examined. FES-of-decentralized-SCG induced basal PAME and SAME release and basal aortic dilation (Fig. $4 A$ and $B$ ), which were not affected by NLA ( $0.3 \mathrm{mM})$ applied onto the SCG (Fig. $4 B)$. In contrast, basal aortic dilation (Fig. $4 C$ and $D$ ) and release of PAME and SAME (Fig. 4E) from denervated SCG upon FES were significantly enhanced by NLA applied onto the SCG (Fig. $4 C$ and D) like that found in the normal control SCG (Fig. 1). These results clearly indicate that PAME and SAME are synthesized and released in the preganglionic neurons of the SCG.

The preganglionic terminals of the rat SCG contain functional $\alpha 7$-nicotinic acetylcholine receptors ( $\alpha 7$-nAChRs) (25) that are involved in regulating transmitter acetylcholine (ACh) release (25). Nicotine $(100 \mu \mathrm{M})$, which binds $\alpha 7-\mathrm{nAChR}$ located on the rat SCG $(26,27)$ to induce calcium influx into neurons $(26,27)$ and generation of inward currents (10), superfused onto the SCG caused aortic relaxation with time course similar to that induced by FES of the SCG (Fig. 1H). This nicotine-induced aortic relaxation was increased significantly by NLA superfused onto the SCG (Fig. $1 H$ and $I$ ). These results suggest that similar to FES, nicotine causes release of PAME from the preganglionic neurons of the SCG and that release of PAME depends on the neuronal calcium influx and depolarization of the SCG neurons.

The calcium dependence of NLA-induced basal release and NLAenhanced FES-elicited release of both FAMEs from the SCG (Fig. 3A) was supported by the findings that release of both FAMEs was drastically diminished in calcium-free Krebs' solution (Fig. 4F). Furthermore, upon superfusion with normal Krebs' solution, NLA enhancement of PAME and SAME release from the SCG upon FES was reduced to basal levels by ML-9 $(30 \mu \mathrm{M})$, a myosin light chain kinase (MLCK) inhibitor that inhibits calcium/calmodulin-dependent (28) synaptic vesicle pool mobilization (29) (Fig. 4G). ML-9 did not affect the basal release of both FAMEs from the SCG upon FES, suggesting that ML-9 at this concentration is specific for inhibiting the active release of FAMEs. ML-9, however, has been shown to inhibit various protein kinases (30). The possibility that release of FAME via nonvesiculardependent mechanisms, however, cannot be excluded. Nonetheless, our present results strongly favor that release of PAME and SAME in the SCG upon depolarization in the presence of arginine analogs is associated with axoplasmic vesicular function.

Accordingly, both FAMEs are likely to be transmission mediators or modulators in the SCG. SA, PA, and other saturated fatty acids with $<20$ hydrocarbon chains have been shown to potentiate ACh-induced, $\alpha 7$-nAChR-mediated inward currents, leading to facilitation of hippocampal neurotransmission (31). The postganglionic neurons/cell bodies of the rat SCG also are endowed with $\alpha 7$-nAChR $(10,25,27)$. Whole-cell patch-clamp studies, however, demonstrated that PAME (Fig. 5A) and PA (Fig. $5 C$ ), but not SAME (Fig. $5 B$ ) or SA (Fig. $5 D$ ), without inducing changes in membrane potential, inhibited nicotine-induced peak and steadystate inward currents (Fig. $5 E$ and $F$ ). These results suggest that PAME and PA may inhibit $\alpha 7$-nAChR-mediated inward currents in the SCG. Similar inhibitory effects of PAME (Fig. $6 A$ and $B$ ) and PA (Fig. $6 C$ ) were found in $\alpha 7$-nAChR-expressing Xenopus oocytes. It is suggested that presynaptically released PAME may negatively modulate the presynaptic $\alpha 7-n A C h R$ and the postsynaptic $\alpha 7$-nAChR on cell bodies of the SCG. The exact reason(s) for differences in findings from that reported by Ohta et al. (31) is not known. In addition to the different tissues used, Ohta et al. (31) did not describe the methods for preparation and delivery of palmitic acid, therefore making it difficult to compare. Our results suggesting that PAME inhibition of inward currents and induction of relaxation, however, are consistent, at least, when calcium mechanisms in the neurons and the smooth muscle cells are concerned.

PAME also is released in the rat parasympathetic sphenopalatine ganglion to cause aortic dilation (H.W.L. and T.J.-F.L., unpublished data). PAME appears to be physiologically important in modulating the autonomic ganglionic transmission. In this regard, the signals conducted in nerves are quick with short half-lives, enabling a quick transmission of the subsequent impulses. The vasodilating effect of PAME will increase the blood flow in the SCG. This effect of PAME lasts longer and may help to remove the released neurotransmitters and modulators, and is expected to accelerate the termination of neurotransmission in normal physiology, a phenomenon in line with PAME blockade of the nicotinic receptor-induced inward currents in SCG neurons. A similar observation is expected for ACh, which may cause vasodilation (5) and increase blood flow in the SCG. Unlike PAME, ACh stimulates the nicotinic receptor on the ganglionic neurons of the SCG $(10,32)$. The exact mechanisms for this important and complicated modulation of ganglionic transmission participated by the transmitter and modulator remain to be examined. The vasodilatory mechanism, however, may facilitate the fast command-response.

The physiological significance of the actively released PAME and SAME may not be limited to the autonomic ganglion. Normal nonischemic brain free fatty acids consist mainly of PA, SA, and oleic acid with smaller amounts of arachidonic acid and others (33), although the highly likely presence of methyl ester forms has not been reported. It is 
A

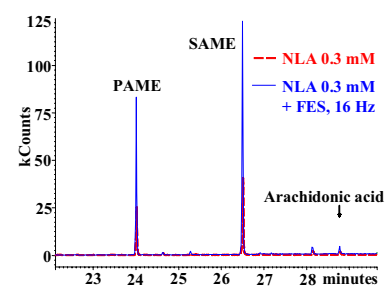

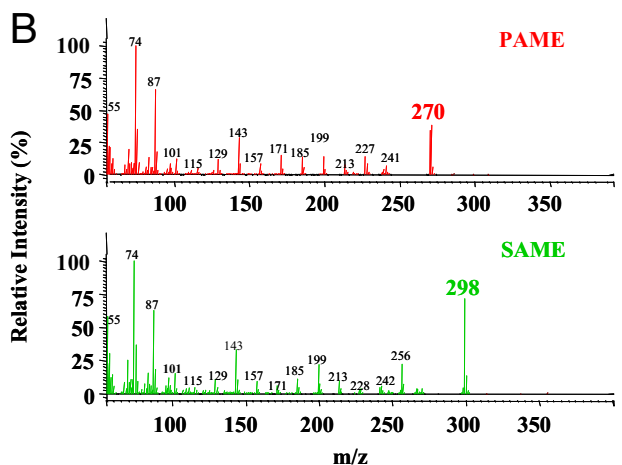

m/

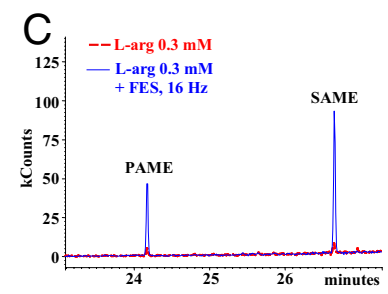

$\mathrm{F}$

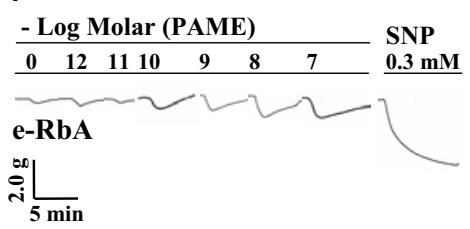

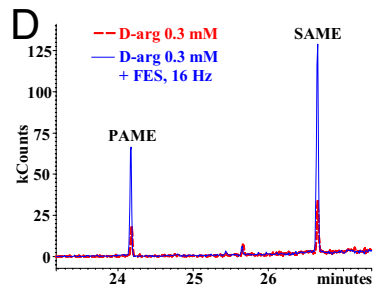
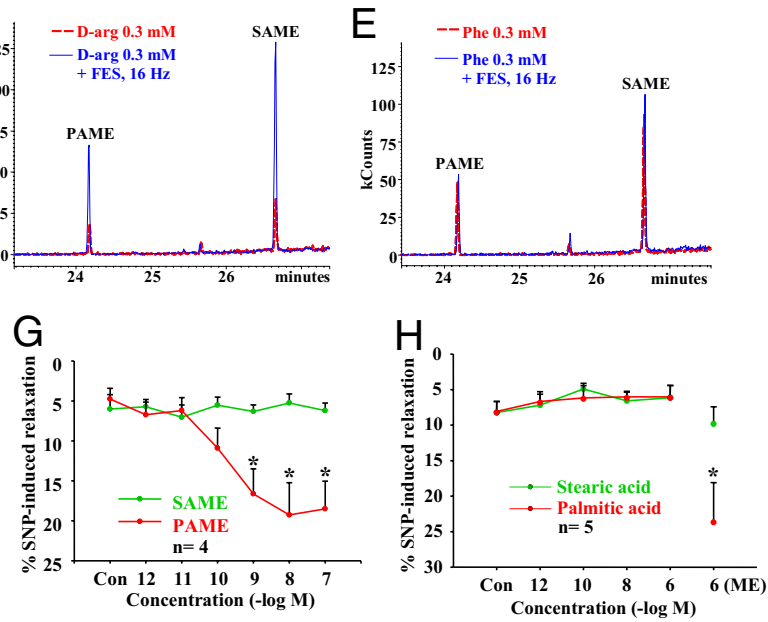

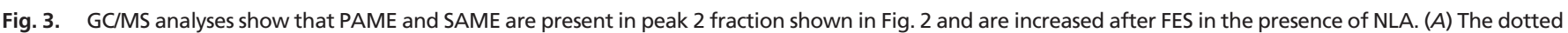

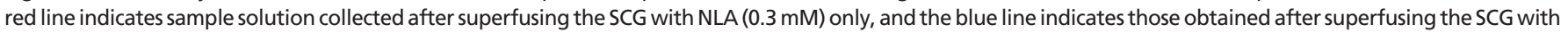

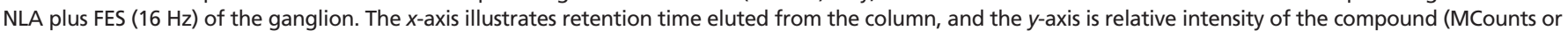

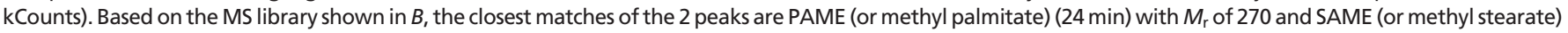

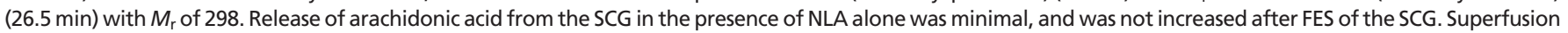

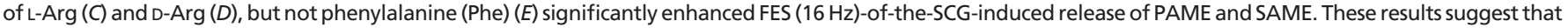

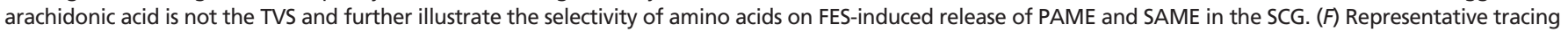

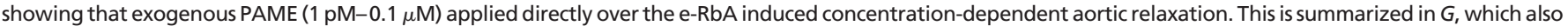

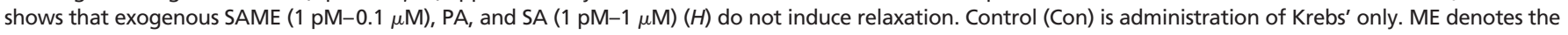
presence of methyl ester in the fatty acid. *, $P<0.05$ denotes significantly different from FES only ( $n=3-5$ for all experiments).

plausible based on present findings that application of NLA may increase regional cerebral blood flow via release of PAME. General findings in normal experimental animals, however, indicate that NOS inhibitors such as NLA attenuate cerebral vasodilation and cerebral blood flow (34). This is caused largely by inhibition of synthesis of endothelial NO, which has higher efficacy than that of PAME in inducing aortic relaxation (present findings). Systemic administration of NLA, however, dilates pial arterioles of endothelial NOS knockout mice and significantly decreases cerebral occlusion infarct size (35). This may be explained by the vasodilatory effect of released PAME from neurons following NLA administration. In addition, it is interesting to note that activity of the uncoupling protein 2 , an inducible protein that is neuroprotective, is enhanced by PA in isolated mitochondria (36).

The present finding provides strong evidence for release of PAME and SAME from the axoplasmic vesicular stores in the preganglionic neurons of the SCG upon electrical and chemical depolarization. The release is significantly enhanced in the presence of arginine analogs and basic amino acids, and is calcium- and MLCK-dependent. PAME negatively modulates nicotinic receptor-mediated ganglionic transmission and is likely to be the most potent known vasodilator. These findings provide a concept for saturated fatty acids and their potential interactions with amino acids in physiological regulation of not only neural transmission but also vascular function.

\section{Materials and Methods}

Tissue Preparation. All experimental procedures were approved by the laboratory animal care and use committee (Southern Illinois University School of Medicine). The male Sprague-Dawley rats (50-100 g) were anesthetized with pentobarbital (35 mg/kg, i.p.). The SCG were removed, placed in Krebs' solution, and the connective capsule of the ganglia was removed (4). Male New Zealand White rabbits (2-3 kg) were anesthetized with pentobarbital (40 mg/kg, i.v.), and the thoracic aorta was removed and cleaned of adventitial adipose tissue. Endothelial cells of the aortic rings were mechanically denuded to eliminate any possible influence of vasodilating substances released from the endothelium (8). The successful removal of endothelial cells was verified by the lack of acetylcholine (3 $\mu \mathrm{M}$ )-induced relaxation (8).

Superfusion Bioassay Cascade. A modified superfusion bioassay cascade system (8) was used. Four SCGs from 2 rats, serving as the donor tissue, were mounted vertically between 2 platinum electrodes in a double-walled glass chamber coated with Sigmacote (Sigma-Aldrich) and superfused $(2.5 \mathrm{~mL} /$ min) with oxygenated Krebs' buffer solution at $37{ }^{\circ} \mathrm{C}$ via a peristaltic pump. The superfusate was allowed to superfuse an isolated e-RbA ( $3 \mathrm{~mm}$ long) as detector tissue arranged in a cascade equilibrated for 60 min under $2 \mathrm{~g}$ of muscle tension. The e-RbA was used as detector tissue because the rabbit aorta contains robust vascular smooth muscle and abundant $\alpha$-adrenoceptors readily producing consistent vasoconstriction in the presence of Lphenylephrine $(0.1 \mu \mathrm{M})$. In addition, e-RbA was superfused $(2.5 \mathrm{~mL} / \mathrm{min})$ directly from a separate line as needed. FES impulses (biphasic square waves, $300 \mathrm{~mA}, 0.3-\mathrm{ms}$ half-wave duration with a pulse train lasting for $60 \mathrm{~s}$ ) 


\section{A Decentralized SCG}

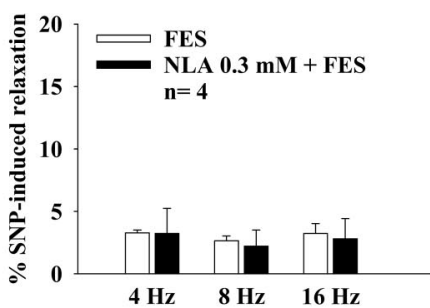

B Decentralized SCG

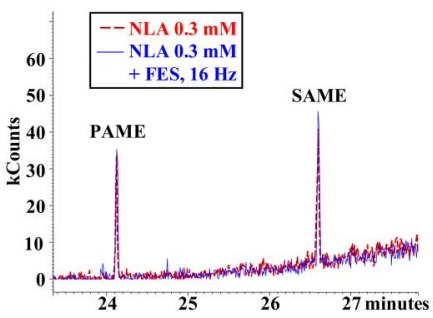

C Denervated SCG

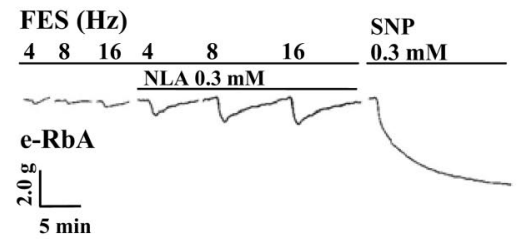

Denervated SCG

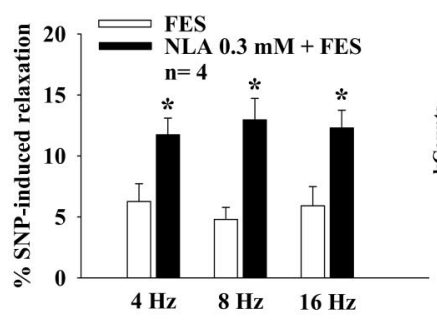

E Denervated SCG

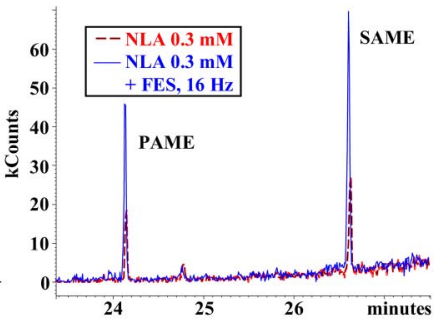

$\mathrm{F} \mathrm{Ca}^{2+}$ free Krebs' solution

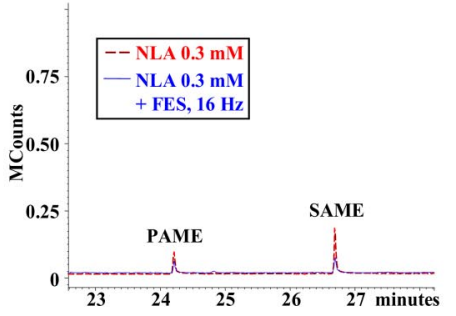

G Normal Krebs' solution

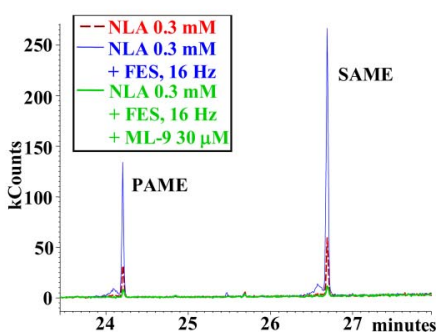

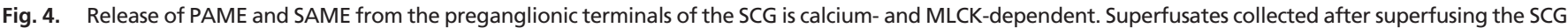

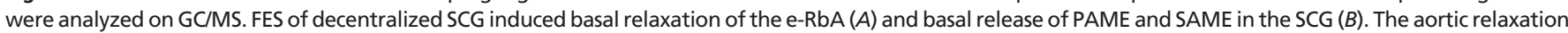

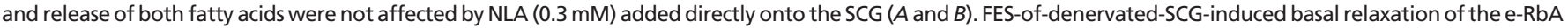

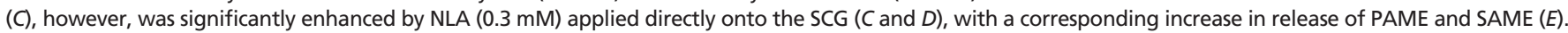
Release of both fatty acids from the normal SCG upon FES in the presence or absence of NLA was drastically decreased when calcium was removed from the Krebs'

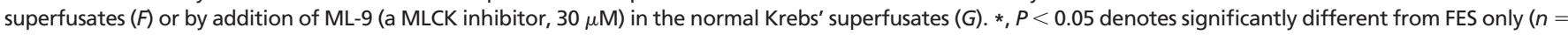
4 for all experiments).

were applied to the SCG (8). The exact parameters for FES were constantly monitored by a Tektronix oscilloscope. Tension changes in the aortic rings were measured by using an isometric transducer (FT03C; Grass) and recorded on a Grass polygraph. Superfusion Krebs' buffer contained L- phenylephrine $(0.1 \mu \mathrm{M})$ to precontract the e-RbA. Atropine $(1 \mu \mathrm{M})$ and guanethidine $(0.3 \mu \mathrm{M})$ were added in the Krebs' superfusate to eliminate the possible influences of cholinergic and adrenergic components, respectively $(37,38)$. Our preliminary results, however, indicated that these
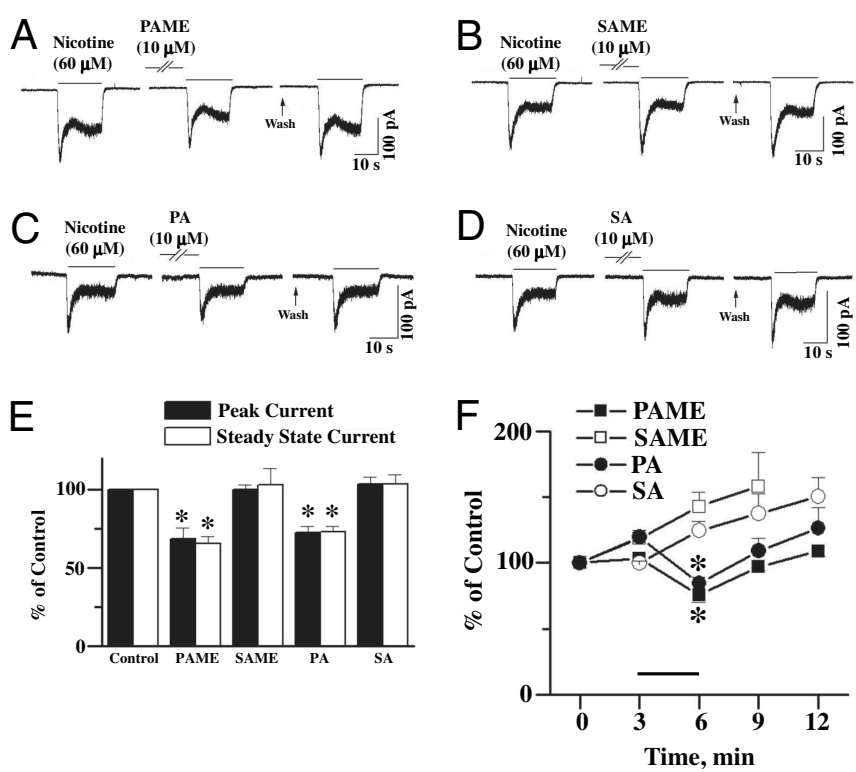

Fig. 5. Nicotine $(60 \mu \mathrm{M})$-induced whole-cell peak and steady-state inward currents in cultured SCGs were inhibited by PAME $(10 \mu \mathrm{M})(A)$ and PA $(10 \mu \mathrm{M})(C)$ but were not affected by SAME $(10 \mu \mathrm{M})(B)$ or SA $(10 \mu \mathrm{M})(D)$. The results are summarized in $E$. They are further summarized in $F$, indicating that whole-cell inward currents increase with repeated applications of nicotine. The inhibition by PAME and PA incubation is reversed after washing off these fatty acids. The horizontal line above the $x$-axis denotes the incubation of FAMEs or fatty acids for $3 \mathrm{~min}$. * denotes significant difference from control $(P<0.01)(n=4)$.
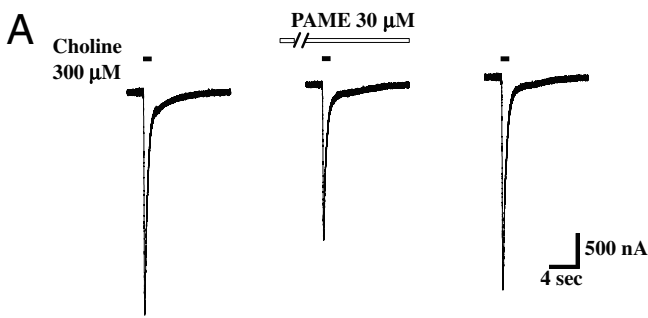

B
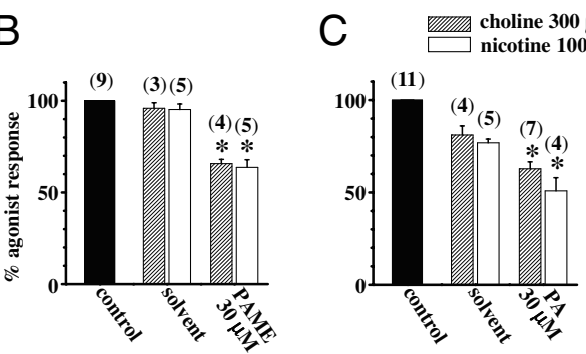

Fig. 6. Effects of PAME and PA on nicotine- and choline-induced inward currents in $\alpha 7$-nAChR-expressing Xenopus oocytes were examined. The resting membrane potentials were not altered by the administration of PAME or PA (30 $\mu \mathrm{M})$. (A) Representative tracings show the inhibitory effect of PAME (30 $\mu \mathrm{M})$ on choline-induced inward currents. Short horizontal solid bars above the tracing denote application of choline for $1 \mathrm{~s}$. Oocytes were continuously perfused with PAME for 3 min indicated by the long horizontal open bar. ( $B$ and $C$ ) Summaries of effects of PAME $(B)$ and PA $(C)$ on nicotine- or choline-induced peak inward currents. The number in parentheses above each column represents numbers of oocytes examined. *, $P<0.05$ indicates significantly different from the solvent group. Values are means \pm SEM. 
antagonists resulted in no difference in basal tone or relaxation of the e-RbA. Therefore, throughout the entire course of these experiments, only L-phenylephrine $(0.1 \mu \mathrm{M})$ was added to the Krebs' superfusate to precontract the e-RbA. At the end of each experiment, SNP $(0.3 \mathrm{mM})$ was added to induce maximal relaxation of the detector aortic ring. Drugs were dissolved in Krebs' solution, and the SCG was superfused for $30 \mathrm{~min}$ followed by washing off of these drugs with prewarmed fresh Krebs' solution for $1 \mathrm{~h}$ via peristaltic pump. Aortic relaxations were estimated as the percentage of maximal relaxation induced by SNP $(0.3 \mathrm{mM})$ and were expressed as mean $\pm \mathrm{SEM}$. $\mathrm{EC}_{50}$ values (39) (the concentration that produces $50 \%$ of the maximum relaxation) were determined by measuring peak vasodilation of the e-RbA for PAME and SNP-induced relaxation of the e-RbA. From these values, the geometric means $\mathrm{EC}_{50}$ values (39) with $95 \%$ confidence intervals were calculated.

Superfusate containing the released substances from the SCG were collected during FES and $1 \mathrm{~min}$ after FES. This yielded $5 \mathrm{~mL}$ of superfusate per stimulation. The superfusates from 4 stimulations were collected, pooled, dehydrated, pelleted, and stored at $20^{\circ} \mathrm{C}$ in the dark for future analyses. In some studies, experimental drugs were applied directly onto the e-RbA.

RP-HPLC. Lyophilized samples were reconstituted with solvent A ( $0.1 \%$ trifluoroacetic acid/trifluoroacetic acid in water) and analyzed by RP-HPLC on a Zorbax 300SB-C18 column $(4.6 \times 250 \mathrm{~mm})$ with an Agilent $1100 \mathrm{HPLC}$ system (Agilent Technologies) at a flow rate of $1.00 \mathrm{~mL} / \mathrm{min}$. Chromatography was carried out with a linear gradient from $0 \%$ to $60 \%$ of solvent B (20\% water and $80 \%$ acetonitrile containing $0.1 \%$ trifluoroacetic acid) for $40 \mathrm{~min}$, and the UV absorption was monitored at $280 \mathrm{~nm}$.

GC/MS. The crude extracts from the superfusion bioassay cascade and RP-HPLC peak sample extracts were methanol $(2 \mathrm{~mL})$ extracted to solubilize the organic compounds. The sample was vortexed, sonicated, and finally pelleted via centrifugation at $151 \times g$ for $5 \mathrm{~min}$ at $20^{\circ} \mathrm{C}$. The supernatant was transferred to $12-\times$ 32-mm screw-cap tubes with polytetrafluoroethylene/silicone septa in the caps. PAME $(1 \mathrm{ng} / \mu \mathrm{L})$ was used as a standard reference. Samples were analyzed by

1. Lee TJF, Su C, Bevan JA (1976) Neurogenic sympathetic vasoconstriction of the rabbit basilar artery. Circ Res 39:120-126.

2. Araki H, Su C, Lee TJF (1982) Effect of superior cervical ganglionectomy on the sensitivity of rabbit ear artery and cerebral arteries of rabbit and cat to vasoactive agents. J Pharmacol Exp Ther 220:49-55.

3. Edvinsson L, Hamel E (2002) in Cerebral Blood Flow and Metabolism, eds Edvinsson L, Krause DN (Lippincott Williams \& Wilkins, Philadelphia), 2nd Ed, pp 43-67.

4. Dun NJ, Dun SL, Wu SY, Forstermann U (1993) Nitric oxide synthase immunoreactivity in rat superior cervical ganglia and adrenal glands. Neurosci Lett 158:51-54.

5. Furchgott RF, Zawadzki JV (1980) The obligatory role of endothelial cells in the relaxation of arterial smooth muscle by acetylcholine. Nature 288:373-376.

6. Briggs CA (1992) Potentiation of nicotinic transmission in the rat superior cervical sympathetic ganglion: effects of cyclic GMP and nitric oxide generators. Brain Res 573:139-146.

7. Palmer RM, Ferrige AG, Moncada S (1987) Nitric oxide release accounts for the biological activity of endothelium-derived relaxing factor. Nature 327:524-526.

8. Chen FY, Lee TJF (1993) Role of nitric oxide in neurogenic vasodilation of porcine cerebral artery. J Pharmacol Exp Ther 265:339-345.

9. Wang Q, Cwik M, Wright CJ, Cunningham F, Pelligrino DA (1999) The in vivo unidirectional conversion of nitro-D-arginine to nitro-L-arginine. J Pharmacol Exp Ther 288:270-273.

10. Long C, et al. (2006) Monoamine uptake inhibitors block $\alpha 7$-nAChR-mediated cerebral nitrergic neurogenic vasodilation. Am J Physiol 291:H202-H209.

11. Blondeau N, et al. (2007) Polyunsaturated fatty acids are cerebral vasodilators via the TREK 1 potassium channel. Circ Res 101:176-184.

12. Gilbert P, Tremblay J, Thorin E (2001) Endothelium-derived endothelin-1 reduces cerebral artery sensitivity to nitric oxide by a protein kinase C-independent pathway. Stroke 32:2351-2355.

13. Shelkovnikov S, Merlic CA, Gonick HC (2004) Influence of nitric oxide donors and peroxynitrite on the contractile effect and concentration of norepinephrine. Life SCi 74:2919-2928.

14. Feelisch M, Kotsonis $P$, Siebe J, Clement B, Schmidt HH (1999) The soluble guanylyl cyclase inhibitor $1 \mathrm{H}-[1,2,4]$ oxadiazolo[ $4,3,-$ a] quinoxalin-1-one is a nonselective heme protein inhibitor of nitric oxide synthase and other cytochrome P-450 enzymes involved in nitric oxide donor bioactivation. Mol Pharmacol 56:243-253.

15. Wiener HL, Thalody GP, Maayani S (1993) Interactions between responses mediated by activation of adenosine $\mathrm{A} 2$ receptors and $\alpha_{1}$-adrenoceptors in the rabbit isolated aorta. Br J Pharmacol 109:394-404.

16. Ansari HR, Nadeem A, Talukder MA, Sakhalkar S, Mustafa SJ (2006) Evidence for the involvement of nitric oxide in A2B receptor-mediated vasorelaxation of mouse aorta. Am J Physiol 292:H719-H725.

17. Pratt PF, Li P, Hillard CJ, Kurian J, Campbell WB (2001) Endothelium-independent, ouabain-sensitive relaxation of bovine coronary arteries by EETs. Am J Physiol 280:H1113-H11121.

18. Pratt PF, Medhora M, Harder DR (2004) Mechanisms regulating cerebral blood flow as therapeutic targets. Curr Opin Investig Drugs 5:952-956.

19. Kaide Jl, et al. (2001) Carbon monoxide of vascular origin attenuates the sensitivity of renal arterial vessels to vasoconstrictors. J Clin Invest 107:1163-1171.

20. Edvinsson L, Ekman R, Jansen I, Ottosson A, Uddman R (1987) Peptide-containing nerve fibers in human cerebral arteries: Immunocytochemistry, radioimmunoassay, and in vitro pharmacology. Ann Neurol 21:431-437. using a Varian Saturn II ion trap GC/MS. The GC, a Varian 3400, was equipped with an RTX-5MS 30-m $\times 0.25-\mathrm{mm}$ (inner) diameter column with a $0.25-\mu \mathrm{m}$ film thickness. Helium was used as the carrier gas, and $4 \mu \mathrm{L}$ of sample was injected by using a splitless injection mode. The injection port was set at $290^{\circ} \mathrm{C}$. The initial column temperature was $40^{\circ} \mathrm{C}$ and was held for $2.5 \mathrm{~min}$. The temperature was then ramped at $8{ }^{\circ} \mathrm{C}$ per min to $250^{\circ} \mathrm{C}$ and then ramped at $20^{\circ} \mathrm{C}$ per min to $310^{\circ} \mathrm{C}$, where it was held for $1 \mathrm{~min}$. The mass spectrometer was set to scan from $55 \mathrm{~m} / \mathrm{z}$ to $310 \mathrm{~m} / \mathrm{z}$ at $0.58 \mathrm{~s}$ per scan. Data were collected and processed by using the Saturn $5.51 \mathrm{GC} / \mathrm{MS}$ Workstation software.

Chemicals and solutions. Details of chemicals and solutions used are found in supporting information (SI) Text .

Decentralization and denervation of the superior cervical ganglion. Detailed methods of denervation (2) are found in SI Text.

Catecholamine fluorescence. Demonstration of cerebral perivascular sympathetic nerves was carried according to our previous report (2). Detailed methods are found in SI Text.

SCG cell culture.Detailed methods of culturing SCG neuronal cells $(10,32)$ are found in SI Text.

Electrophysiology. Detailed methods of patch-clamp technique to record whole-cell currents $(10,32)$ of SCG neuronal cells and 2-electrode voltage clamp for $\alpha 7$-nAChR-expressing oocytes (27) are found in SI Text.

Statistics. Results are expressed as means \pm SEM. Statistical analysis was evaluated by Student's $t$ test for paired or unpaired samples as appropriate. The $P<0.05$ level of probability was accepted as significant.

ACKNOWLEDGMENTS. We thank G. Bordson and A. Harms for assistance with GC/MS, RP-HPLC, and LC/MS; J. Holcomb and the research services staff at Southern Illinois University School of Medicine for fabrication of equipment needed for this work; H. Suchy and D. Born for assistance with surgical operations; and E. Chou and S. Tischkau for reviewing the article. This work was supported by National Institutes of Health Grants HL 27763 and HL 47574; the Southern Illinois University School of Medicine; Tzu-Chi University Grant TCMRC-C95005-01; and National Science Council of Taiwan Grants NSC-92-2320-B-320-025, 93-2745-B320-004-URD, NSC-95-2320-B-320-013-MY2, and NSC-96-2320-B-320-005-MY3.

21. Lee TJ, Saito A, Berezin I (1984) Vasoactive intestinal polypeptide-like substance: The potential transmitter for cerebral vasodilation. Science 224:898-901.

22. Saito A, Masaki T, Uchiyama Y, Lee TJ, Goto K (1989) Calcitonin gene-related peptide and vasodilator nerves in large cerebral arteries of cats. J Pharmacol Exp Ther 248:455462.

23. Chunhabundit $P$, Thongpila S, Somana R (1992) Microvascularization of the rat superior cervical ganglion: A 3-dimensional observation. Acta Anat 143:54-58.

24. Purves D, Lichtman JW (1978) Formation and maintenance of synaptic connections in autonomic ganglia. Physiol Rev 4:821-862.

25. Liang SD, Vizi ES (1997) Positive feedback modulation of acetylcholine release from isolated rat superior cervical ganglion. J Pharmacol Exp Ther 280:650-655.

26. Si ML, Lee TJF (2002) $\alpha 7$-Nicotinic acetylcholine receptors on cerebral perivascular sympathetic nerves mediate choline-induced nitrergic neurogenic vasodilation. Circ Res 91:62-69.

27. Mozayan M, et al. (2006) Cholinesterase inhibitor blockade and its prevention by

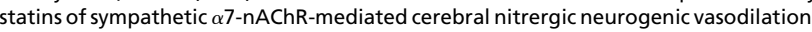
J Cereb Blood Flow Metab 26:1562-1576.

28. Sellers JR, Pato MD (1984) The binding of smooth muscle myosin light chain kinase and phosphatases to actin and myosin. J Biol Chem 259:7740-7746.

29. Ryan TA (1999) Inhibitors of myosin light chain kinase block synaptic vesicle pool mobilization during action potential firing. J Neurosci 19:1317-1323.

30. Bain J, McLauchlan H, Elliott M, Cohen P (2003) The specificities of protein kinase inhibitors: An update. Biochem J 371:199-204.

31. Ohta K, et al. (2003) Stearic acid facilitates hippocampal neurotransmission by enhancing nicotinic ACh receptor responses via a PKC pathway. Brain Res Mol Brain Res 119:83-89.

32. Mozayan M, Lee TJ (2007) Statins prevent cholinesterase inhibitor blockade of sympathetic $\alpha 7$-nAChR-mediated currents in rat superior cervical ganglion neurons. Am Physiol 293:H1737-H1744.

33. Yasuda H, Kishiro K, Izumi N, Nakanishi M (1985) Biphasic liberation of arachidonic and stearic acids during cerebral ischemia. J Neurochem 45:168-172.

34. Zhang F, Xu S, ladecola C (2005) Role of nitric oxide and acetylcholine in neocortical hyperemia elicited by basal forebrain stimulation: Evidence for an involvement of endothelial nitric oxide. Neuroscience 69:1195-1204.

35. Huang Z, et al. (1996) Enlarged infarcts in endothelial nitric oxide synthase knockout mice are attenuated by nitro-L-arginine. J Cereb Blood Flow Metab 16:981-987.

36. Mattiasson G, et al. (2003) Uncoupling protein-2 prevents neuronal death and dimin ishes brain dysfunction after stroke and brain trauma. Nat Med 9:1062-1068.

37. Lee TJF, Kinkead LR, Sarwinski S (1982) Norepinephrine and acetylcholine trans mitter mechanisms in large cerebral arteries of the pig. J Cereb Blood Flow Metab 2:439-450.

38. Moummi C, Gullikson GW, Gaginella TS (1992) Effect of endothelin-1 on guinea pig gallbladder smooth muscle in vitro. J Pharmacol Exp Ther 260:549-553.

39. Fleming WW, Westfall DP, De La Lande JS, Jellett LB (1972) Log-normal distribution of equieffective doses of norepinephrine and acetylcholine in several tissues. $J$ Pharmaco Exp Ther 181:339-345. 